\title{
Fever of Unknown Origin: Don't Forget Kala-Azar Even in Europe
}

\author{
Javier Moreno Díaz ${ }^{1}$, David de las Cuevas León², Ana Martínez González ${ }^{3}$, Rebeca Rubio Escuin ${ }^{4}$ \\ ${ }^{1}$ Internal Medicine Department, Hospital Universitario Miguel Servet, Zaragoza, Spain \\ ${ }^{2}$ Cardiology Department, Hospital Universitario Miguel Servet, Zaragoza, Spain \\ ${ }^{3}$ Rehabilitation Department, Hospital de Barbastro, Huesca, Spain \\ ${ }^{4}$ Haematology Department, Hospital Universitario Miguel Servet, Zaragoza, Spain
}

Received: 30/10/2017

Accepted: 20/11/2017

Published: 06/12/2017

How to cite this article: Moreno J, de las Cuevas D, Martínez AM, Rubio R. Fever of unknown origin: dont' forget Kala-Azar even in Europe. EJCRIM 2017;4: doi:10.12890/2017_000772.

Conflicts of Interests: The Authors declare that there are no competing interests.

This article is licensed under a Commons Attribution Non-Commercial 4.0 License

\section{ABSTRACT}

We present the case of a 70-year-old male patient with fever of unknown origin after a long period of convalescence from a previous admission to a chronic care hospital. During the admission, multiple combinations of antibiotic and antifungal treatments were prescribed, but with persistent fever and, eventually, neutropenia (200 lymphocytes, 0 neutrophils). Given the suspicion of infection at bone marrow level, a biopsy was performed as was serology of Leishmania, both diagnostic determinations. Treatment with amphotericin B liposomal resulted in a good outcome.

\section{LEARNING POINTS}

- Visceral leishmaniasis is a rare aetiology of neutropenia, which causes an important constitutional syndrome and long-standing fever.

- However, this pathology is endemic in areas of the Mediterranean Sea, which is well known for migratory flows.

- High clinical suspicion of leishmaniasis is necessary in patients with fever of long evolution, hepatomegaly or splenomegaly, fever and constitutional syndrome, and from endemic areas.

\section{KEYWORDS}

Leishmania, leishmaniasis, neutropenia, fever, kala-azar

\section{INTRODUCTION}

Visceral leishmaniasis is a disease caused mainly by Leishmania donovani and Leishmania infantum. The main reservoirs in our environment are dogs, although mice and other wild animals can also transmit the flagellated protozoan through the bite of the female sandfly. In Spain, there have been relatively common outbreaks of Leishmania, such as that which occurred in the Community of Madrid between 2009 and 2012. Its incidence in our environment is 0.45 cases per 100,000 inhabitants. This is because the disease is present endemically in areas of the Mediterranean, more specifically in rural areas, mountain towns and peri-urban regions. 


\section{CASE DESCRIPTION}

A 70-year-old Spanish man with a history of hypertension, ex-smoker, ischaemic cardiopathy and chronic heart failure with left ventricular ejection fraction (LVEF) in the mid-range, severe chronic obstructive pulmonary disease (COPD), and chronic atrial fibrillation was admitted to our hospital from the emergency room due to sepsis of probable respiratory origin with acute renal failure and secondary digital toxicity, together with pancytopenia. He received combination antibiotic treatment of meropenem with amikacin and daptomycin, as well as fluconazole, later simplified to piperacillin/tazobactam, in spite of which the patient continued to have asymptomatic febrile peaks.

Serial blood cultures were negative. Following haematology consultation for pancytopenia, probable myelodysplastic syndrome was diagnosed, without performing a core biopsy. After respiratory stabilisation and slight improvement of the functional class, the patient was transferred to a convalescent hospital for functional recovery and rehabilitation.

He remained in the convalescence hospital for 3 months, however, with progressive worsening of his general condition and persistent fever, for which the antibiotics received in the tertiary hospital were progressively reintroduced. Finally, he was transferred to our hospital due to persistent high fevers of 5 consecutive days.

On examination this time, he presented with intense sarcopenia together with poor general condition; cardiopulmonary exploration was normal, without evidence of visceromegaly in abdominal exploration. A clinical ultrasound was performed which showed an estimated LVEF of $45 \%$, moderate mitral insufficiency, absence of pericardial or pleural effusion, and absence of abdominal visceromegaly.

In one blood test carried out, the presence of 200 lymphocytes with 0 neutrophils was evidenced, as well as severe thrombocytopenia. In the study, serology was performed for HIV, hepatitis C virus (HCV) and hepatitis B virus (HBV), which were all negative, as well as viral load of cytomegalovirus (CMV). Serology for Leishmania was positive, with a median title of 1:2560.

Given these findings, it was decided to perform a sternal bone marrow biopsy. It showed the presence of parasites in bone marrow, taxonomically catalogued as Leishmania infantum (Figs. 1, 2, 3 and 4). The patient started treatment with liposomal amphotericin B with good clinical evolution, improvement of pancytopenia, and resolution of fever.

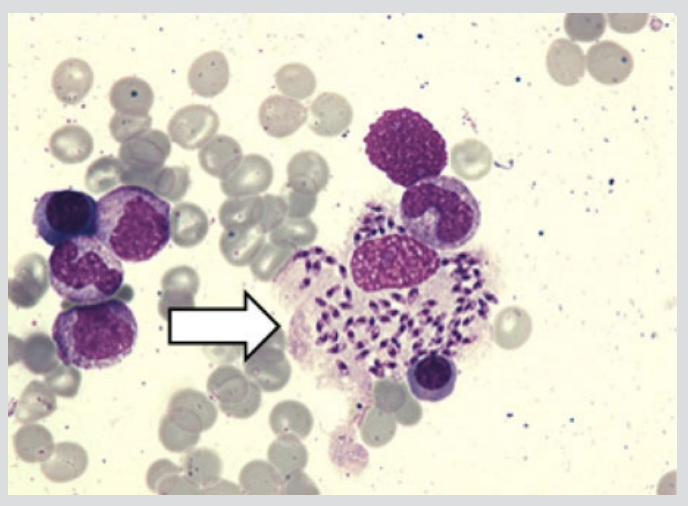

Figure 1. Monocyte-macrophage filled with Leishmania amastigotes phagocytosed in the cytoplasm and surrounded by granulocytes and thrombocytes

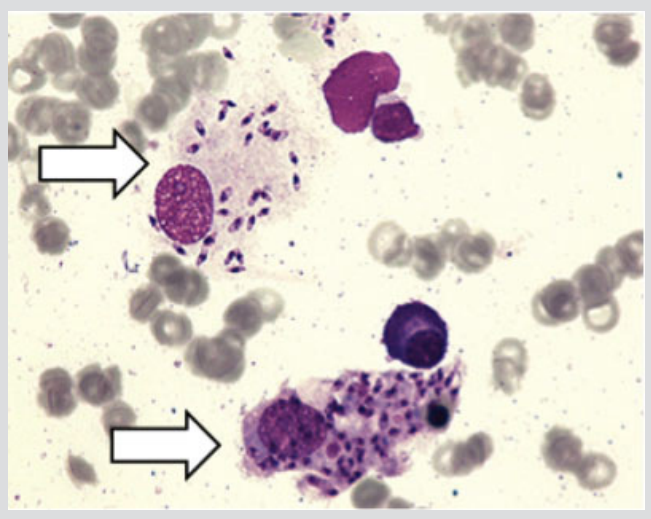

Figure 3. Two cell monocyte-macrophage system with multiple Leishmania amastigotes phagocytosed inside

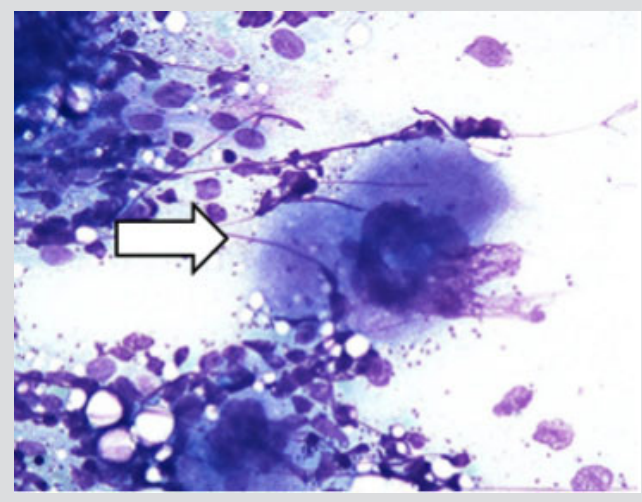

Figure 2. Megakaryocytes surrounded by Leishmania

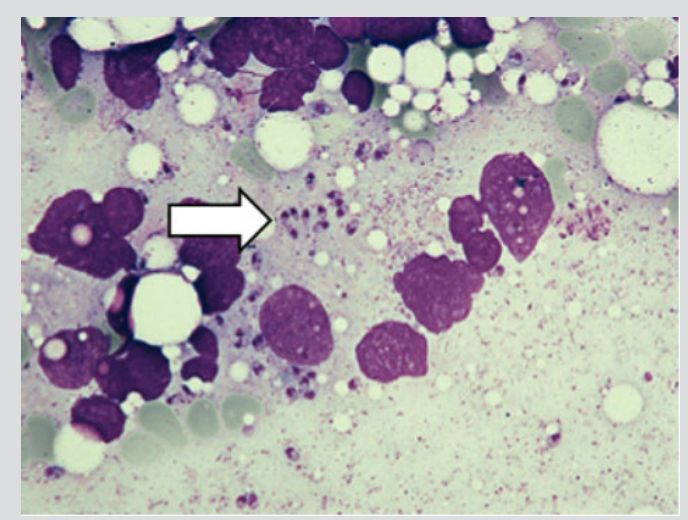

Figure 4. Free Leishmania amastigotes in the interstitial tissue of the bone marrow 


\section{DISCUSSION}

Usually, Leishmania infection is asymptomatic, a consequence of the ability of the immune system to control parasitic replication. In Europe, only 1 in 30 people infected manifest the disease clinically, compared to 1 in 4 people in India, where the disease is endemic. This highlights the differences in virulence among several parasitic strains, genetic predisposition, nutritional status, age, and other factors. Subclinical infection can be evidenced in serological tests, and manifestations can appear before immunosuppression of the individual that contains the parasite in the phagocytic mononuclear system ${ }^{[1,2]}$.

The main clinical manifestation of visceral leishmaniasis is the syndrome known as kala-azar. The incubation period ranges from 2 to 6 months, with symptoms of insidious onset in the form of fever, weight loss, and splenomegaly with or without hepatomegaly. Patients may present with abdominal discomfort, especially in the left hypochondrium. The spleen can be palpated and usually is not painfu[ ${ }^{[3]}$. Anaemia, neutropenia, eosinopenia, and thrombocytopenia are common in laboratory tests.

The main methods to establish the diagnosis of infection include direct visualisation of the parasite in an extension of a tissue sample obtained by biopsy (bone marrow / spleen), culture in special media, and detection by polymerase chain reaction (PCR) of genetic material or serology. However, diagnostic certainty is made by direct observation of the parasite ${ }^{[1,4,5]}$.

The treatment of first choice for visceral leishmaniasis is liposomal amphotericin B $3 \mathrm{mg} / \mathrm{kg} /$ day intravenously in immunocompetent patients and $4 \mathrm{mg} / \mathrm{kg} / \mathrm{day}$ in immunosuppressed patients. Meglumine antimoniate can be used instead, or miltefosine or paromomycin sulfate as a second choice.

\section{REFERENCES}

1. Aronson N, Herwaldt BL, Libman M, Pearson R, Lopez-Velez R, Weina P, et al. Diagnosis and treatment of leishmaniasis: clinical practice guidelines by the Infectious Diseases Society of America (IDSA) and the American Society of Tropical Medicine and Hygiene (ASTMH). Am J Trop Med Hyg 2017;96:24-45.

2. Bogdan C, Rollinghoff M. The immune response to Leishmania: mechanisms of parasite control and evasion. Int J Parasitol 1998;28:121-34.

3. Bern C, Joshi AB, Jha SN, et al. Factors associated with visceral leishmaniasis in Nepal: bed-net use is strongly protective. Am J Trop Med Hyg 2000;63:184-8.

4. Sundar S, Rai M. Laboratory diagnosis of visceral leishmaniasis. Clin Diagn Lab Inmunol 2002;9:951-8.

5. Antinori S, Calattini S, Longhi E, et al. Clinical use of polymerase chain reaction performed on peripheral blood and bone marrow samples for the diagnosis and monitoring of visceral leishmaniasis in HIV-infected and HIV-uninfected patients: a single-center, 8-year experience in Italy and review of the literature. Clin Infect Dis 2007;44:1602-10. 